proper separation of the shadows on a rule at $100 \mathrm{~cm}$. from the pin.

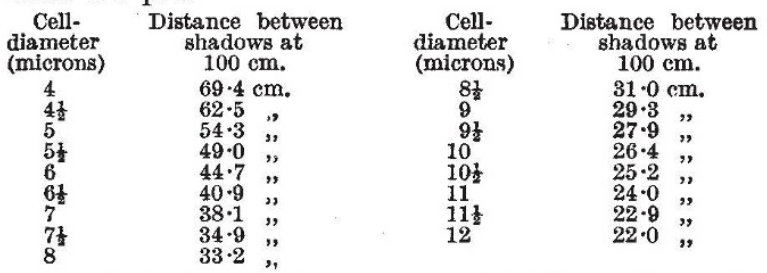

The technique is insert a suitable blood film and, pointing the instrument at a diffuse source of light, to adjust the separation of the coloured rings until the second red rings in each system touch, and then to read off on the scale. It is important that the film be well covered to give a bright spectrum, but there should not be much overlap as the reading represents the predominant diameter of object on the slide (not the arithmetic mean). Two or three films should be examined as a check, approaching the end point from each side in each case. The degree of anisocytosis is represented by the spread of the component spectra, that is, of the spread between the outer red of the smaller cells and the inner violet of the larger cells. In rare cases of anæmia with high degree of anisocytosis the method is completely vitiated by blurring.

No. 1 Field Bacteriological Unit,

Mary Hogben.

Friends' Ambulance Unit H.Q., M.E.F. Dec. 29.

\section{Sorption of Fumigants}

Work recently published by Lubatti $^{1}$ and by Winteringham ${ }^{2}$ and experiments still in progress in the Imperial College seem to give some insight into the mechanism of the sorption of organic vapours, used as fumigants, on wheat.

Fumigants are sorbed by wheat very slowly. Three main factors are involved, (a) permeability of the seed coats, $(b)$ diffusion and sorption in the endosperm, (c) chemical reactions which may take place in the tissues of the grains. The seed coats form a continuous envelope relatively impervious to gases, and they appear to be the main factor controlling sorption. In wheat which has been deprived by abrasion of the seed coats, sorption appears te be of the same order as that in ground wheat.

The investigations of Barrer and Rideal ${ }^{3}$ and those of Wicke ${ }^{4}$ have established that in certain sorbents the rate of sorption is governed by the laws of diffusion. This is true in wheat with vapours which do not undergo a chemical decomposition or are only slightly reactive. Examples of such vapours are those of ethylene dichloride and hydrogen cyanide. Equilibrium with these is substantially reached in 15-20 days, and the experimental curves follow closely those calculated from Fick's law.

The forces which retain ethylene dichloride and hydrogen cyanide in wheat are predominantly physical (van der Waals). Accordingly, sorption decreases with increase in temperature. It has also been found that the amount of fumigant sorbed is approximately the same at any time-concentration product, whether the concentration is kept constant or allowed to fall, provided that the average concentrations are the same.

Other vapours, such as those of ethylene oxide, react with the constituents of the wheat grains. The reaction does not usually take place with one specific chemical compound. Many constituents of the tissues take part in the process, in which enzymic action may also assist. The rate of decomposition of the fumigant in the course of these reactions is apparently unimolecular. This may be due to the slowest process being diffusion through the tissues of the grain. The analogy between diffusion through polymers and unimolecular reactions has been pointed out by Barrer ${ }^{5}$. When sorption of the vapour is accompanied by chemical reaction, equilibrium is not approached even after several days. The curve representing the rate of sorption results from the superposition of two or more curves. With ethylene oxide there is a definite curvature towards the time axis during the first twenty-four hours, while physical forces are predominant; but later the curves straighten out and rise rapidly with time as the chemical action proceeds. It is during this period that the rate of diffusion through the tissues is probably the limiting factor. It would appear that these complex changes cannot be represented by mathematical expressions which would apply to more than one system.

With chemically active vapours, sorption increases with an increase in temperature. The time-sorption curve, calculated from experiments carried out with falling concentration, gives lower values than those obtained in experiments performed at an equivalent even concentration. Benton's work ${ }^{6}$ has shown that the velocity of chemical reactions at constant volume is not the same as that occurring in flow systems.

The sorption isotherms at a steady concentration, within the range of concentrations examined, appear to be rectilinear. As equilibrium is not rapidly reached, the so-called isotherms must be represented by a family of rectilinear curves arranged fanwise, the slope of the curves increasing with time. There appears to be a striking analogy between sorption by wheat and permeation through membranes. The work of Daynes ${ }^{7}$, Barrer ${ }^{5}$ and others has shown that the rate of activated diffusion of gases through membranes of organic polymers is linearly related to the difference of pressure established between the surfaces of the membrane.

An increase of the moisture contents of wheat brings about an increase of sorption with all the fumigants studied. This also may be connected with changes of permeability and structural rearrangements in the tissues of the wheat grain. Moisture content is a factor as important as time, concentration of vapour or temperature. A clearer view of the sorption of vapours by wheat is obtained when the 'isohygrotherms' are represented by tridimensional graphs showing the amount of vapour taken up against the changes of concentration and time.

A fuller account of the experiments referred to in this letter and the conclusions which can be drawn from them will be published elsewhere.

$$
\text { O. F. Lubattr. }
$$

Dept. of Zoology and Applied Entomology, Imperial College of Science and Technology, London, S.W.7. March 8.

${ }^{1}$ Lubatti, J. Soc. Chem. Ind., 63, 257 and 353 (1944).

Winteringham, J. Soc. Chem. Ind., 63, 144 (1944).

s Barrer and Rideal, Proc. Roy. Soc., A, 149, 231 (1935). Barrer, Proc. Roy. Soc., A, 149, 253 (1935).

- Wicke, Koll. Z., 86, 167 (1939).

5 Barrer, "Diffusion in and through Solids" (Cambridge Univ. Press, 1941).

'Benton, Ind. Eng. Chem., 19, 494 (1927) and J. Amer. Chem. Soc., 53, 2984 (1931).

' Daynes, Proc. Roy. Soc., A, 97, 286 (1920). 